\title{
Is There a Secondary Vaccine Hesitancy? Parental Attitudes Toward Revaccination of Childhood Cancer Survivors
}

\author{
Ogochukwu Ezeoke ${ }^{1}$, Sasidhar Goteti ${ }^{2}$, Jamie Cashell ${ }^{3}$, and Amy Caruso-Brown ${ }^{4}$ \\ ${ }^{1}$ Ann and Robert H Lurie Children's Hospital of Chicago \\ ${ }^{2}$ Oregon Health \& Science University \\ ${ }^{3}$ Penn State Health Children's Hospital \\ ${ }^{4}$ State University of New York Upstate Medical University
}

November 23, 2020

\begin{abstract}
Introduction: Many children and adolescents who were vaccinated prior to cancer treatment lose humoral immunity after completion of therapy. Pediatricians and pediatric oncologists often recommend re-immunization, although there is little consensus on timing and approach to serologic testing. However, vaccine hesitancy in the U.S. is a growing problem. It is not known whether parents who initially permitted vaccination might demonstrate secondary hesitancy regarding re-immunization. Methods: We conducted a qualitative study to explore parental attitudes toward re-immunization after completion of cancer therapy. Twenty primary caregivers of current pediatric cancer patients participated in structured interviews exploring knowledge and understanding of immunity and vaccination; previous experiences with vaccines; and attitudes toward vaccines and revaccination. Results: Of those interviewed, $80 \%$ were female and $90 \%$ were White Non-Hispanic. Of interviewees' children with cancer, $60 \%$ were male, $75 \%$ had been diagnosed within the past 6 months, and $45 \%$ had leukemia or lymphoma. All caregivers demonstrated a basic understanding of vaccination, but only $65 \%$ understood that it was possible to lose immunity even with previous vaccination. All caregivers were willing to have their children immunized if tests showed lack of humoral immunity, with $85 \%$ expressing a preference for testing prior to revaccination. Conclusions: Primary caregivers of children with cancer are willing to consider re-immunization interest but do express some secondary hesitancy and strongly prefer that the need for re-immunization be established via serologic testing, rather than performed empirically. Caregivers' beliefs and preferences regarding re-immunization in pediatric oncology should be considered in the development of post-treatment guidelines.
\end{abstract}

\section{Hosted file}

Manuscript - Vaccine Hesitancy.pdf available at https://authorea.com/users/377928/articles/ 494548-is-there-a-secondary-vaccine-hesitancy-parental-attitudes-toward-revaccinationof-childhood-cancer-survivors 


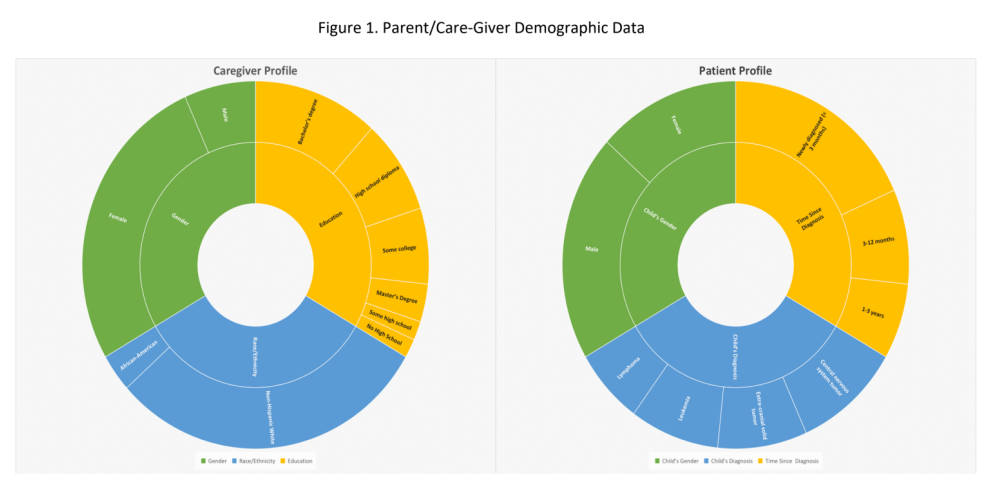

Figure 2. Safety and Choice of Vaccinations

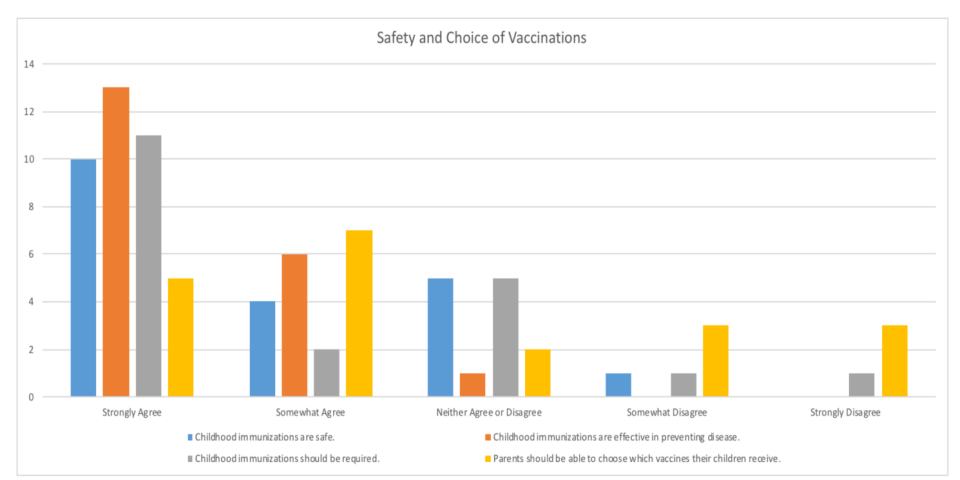

